Anuario Latinoamericano Ciencias Políticas

y Relaciones Internacionales

vol. 10,2020

pp. 55-69

\section{Construcción de la Franja y la Ruta a través del Área de la Gran Bahía de China: oportunidades para América Latina*}

DOI:10.17951/al.2020.10.55-69

\section{Construction of the Belt and Road Through the Greater Bay Area of China. Opportunities for Latin America}

\author{
Daniel Morales Ruvalcaba ${ }^{\star \star}$ \\ CENTER FOR LATIN AMERICAN STUDIES, \\ SUN YAT-SEN UNIVERSITY (中山大学), CHINA \\ $\triangle$ morales@mail.sysu.edu.cn \\ https://orcid.org/0000-0002-4304-3831
}

\section{RESUMEN}

La Provincia de Guangdong concentra su mayor potencial en la llamada Área de la Gran Bahía (AGB). Pero ¿qué representa esta área para América Latina y qué oportunidades existen aquí para la profundización de las relaciones sino-latinoamericanas en el marco de la iniciativa de la Franja y la Ruta (一带一路)? Este trabajo se apoya en los métodos histórico y analítico: el primero para desentrañar el proceso de conformación del $A G B$, el segundo para analizar las potencialidades geográficas, administrativas y organizativas actuales del AGB. El objetivo del trabajo es presentar algunas perspectivas para el desarrollo de los vínculos entre China y América Latina a través del $A G B$ en el marco de la iniciativa de la Franja y la Ruta.

PALABRAS CLAVE: China, América Latina, Área de la Gran Bahía, iniciativa de la Franja y la Ruta (一带一路).

* Este trabajo ha contado con la valiosa colaboración del Mtro. Jiyi Wei y de la estudiante Xiaomin $\mathrm{Xu}$, quienes han contribuido en la recopilación de información, sistematización de datos y búsqueda de notas periodísticas. A ellos mi reconocimiento y agradecimiento.

${ }^{*}$ Doctor en Ciencias Sociales (Universidad de Guadalajara), Maestro en Estudios Contemporáneos de América Latina (Universidad Complutense de Madrid) y Licenciado en Estudios Internacionales (Universidad de Guadalajara). Actualmente es investigador en el Centro de Estudios Latinoamericanos de la Universidad Sun Yat-sen (China) y miembro del Sistema Nacional de Investigadores (México). Ha trabajado como profesor en universidades de México, España, Polonia y China. Además, ha realizado estancias académicas en la Universidad de la República (Uruguay), en el Sciences Po (Francia) y en la Universidad Maria Curie-Skłodowska (Polonia). Como parte de sus labores de investigación, ha publicado diversos artículos científicos sobre Relaciones Internacionales y ha fungido como dictaminador y miembro del comité editorial de varias revistas académicas de América Latina y Europa. Además, es creador del World Power Index (www.worldpowerindex.com). 
Dossier América Latina: la iniciativa china de la Franja y la Ruta

\section{ABSTRACT}

Guangdong Province concentrates its greatest potential in the so-called Greater Bay Area (GBA). But what does this area represent for Latin America and what opportunities exist here for the deepening of Sino-Latin American relations within the framework of the Belt and Road Initiative (一带一路)? This work is based on historical and analytical methods: the first to unravel the process of formation of the GBA, the second to analyze the current geographic, administrative, and organizational potential of the GBA. The objective of the work is to present some perspectives for the development of ties between China and Latin America through the GBA within the framework of the Belt and Road Initiative.

KEYWORDS: China, Latin America, Great Bay Area, Belt and Road Initiative (一带一路).

\section{Introducción}

Más que una nación homogénea y compacta, China es en realidad "una vasta red de vinculaciones humanas, comerciales, y de inversiones entre territorios con diferente estatus jurídico" (Di Masi, 2006, p. 73), sin embargo, a pesar de la creciente literatura sobre las relaciones entre China y América Latina, son prácticamente inexistentes los estudios que aborden, de manera puntual y sistemática, la relación entre los países latinoamericanos con provincias o con zonas específicas de China.

En ese sentido, una de las provincias que amerita especial atención es Guangdong (广东o Cantón). Según Bui, Jones, Li y Yang, "desde la reforma económica de China en 1979, Guangdong, una provincia del sur de China, ha crecido a una tasa anual promedio del $14 \%$, muy superior a la de los Cuatro Pequeños Dragones Asiáticos" (Bui, Jones, Li y Yang, 2003). Efectivamente, en las últimas décadas Guangdong se ha convertido en un relevante caso de estudio, pues no sólo ha funcionado como la auténtica "locomotora" de la economía nacional (con un crecimiento anual promedio de $10,2 \%$ entre 1980 y 2000), sino también una de las provincias precursoras en los esfuerzos de innovación y la apertura de China. Ahora bien, de la totalidad de Guangdong, la zona que más destaca es el Área de la Gran Bahía (AGB). Si bien esta es hoy una de las principales zonas urbanas del mundo, "para el 2030, el PIB del Área de la Gran Bahía se espera que alcance un total de RMB¥30,4 billones (US $\$ 4,62$ billones), sobrepasando el tamaño económico del Área de la Bahía de Tokio (RMB¥ 21,3 billones, US\$3,24 billones) y del Área de la Bahía de Nueva York (RMB¥14,4 billones, US\$2,18 billones), para convertirse en la más grande bahía del mundo en términos de tamaño económico" (PwC, 2017, p. 6).

Cabe destacar que todo el impulso que China está imprimiendo a esta zona no es aislado, sino que se articula con sus Planes de Desarrollo, así como a la iniciativa de la Franja y la Ruta (一带 一 路). Según se establece en la Parte 5 del 13 Plan Quinquenal para el Desarrollo Económico y Social (2016-2020), el gobierno chino ha organizado el desarrollo del país en dos ejes geoestra- 
tégicos: el eje horizontal se extiende a lo largo del río Yangtzé, conectando el interior de China con la megalópolis de Shanghái (en el delta del río Yangtzé), mientras que eje vertical parte del núcleo Beijing-Tianjin-Hebei (Jing-Jin-Ji) $\mathrm{y}$ atraviesa tres provincia de China central-meridional (Henan, Hubei y Hunan) hasta conectar con el Área de la Gran Bahía, zona que reúne el 52 \% de la población cantonesa. En ese sentido, Guangdong y, de manera más específica, el AGB no sólo representan un punto neurálgico del eje vertical de desarrollo, sino también un "portal de China para su apertura" (Central Committee of the Communist Party of China, 2016, p. III, c. 49, sec. 1).

Establecido lo anterior, cabe entonces preguntar: ¿cómo ha sido el proceso de conformación del AGB y cuáles son sus principales potencialidades?, ¿de qué forma se articula el AGB con la iniciativa de la Franja y la Ruta (一带 一路)? Y, en ese sentido, ¿qué representa el AGB para América Latina y qué oportunidades existen para la profundización de las relaciones sinolatinoamericanas desde dicha zona? Para su realización, este trabajo se apoya en el método histórico y en el método analítico: con el primero, se detectan algunas características generales en el proceso histórico de Guangdong y del AGB; a través del segundo, se realiza un análisis de las ciudades que componen el AGB y se mencionan algunas de sus principales potencialidades.

Además de la presente introducción, este trabajo consta de otras cuatro partes: primero se exponen algunas características excepcionales de Guangdong y del AGB; después, se revisa la evolución y situación actual del AGB; posteriormente, se analiza la vinculación que tiene el AGB con los planes de desarrollo interno de China y con su iniciativa de la Franja y la Ruta; y, en la última, se presentan algunas oportunidades para el desarrollo de los vínculos entre China y América Latina a través del AGB.

\section{Excepcionalidad de Guangdong y del Área de la Gran Bahía}

No se puede comprender la importancia del AGB sin dimensionar el peso específico de Guangdong en la historia milenaria y reciente de China. Por ello, en la siguiente sección, se presentan algunos elementos y principales características de dicha provincia.

Si bien los primeros asentamientos cantoneses datan de aproximadamente hace 7000 años, la región comenzó a adquirir cohesión social y política durante el Reino de Nanyue (204-111 a. C.), que tuvo su sede en Panyú, actualmente distrito de Guangzhou. De hecho, las investigaciones arqueológicas ahí realizadas han permitido documentar el aventajamiento agrícola, manufacturero y cultural de dicha zona -gracias a la política nanyue de asimilación y encuentro- así como su temprana vinculación con comercial el mundo, pues se han encontrado materiales provenientes del Sur de Asia, de la actual India e, incluso, de África. Al respecto, Jorge Di Masi explica que el "carácter
Construcción de la Franja y la Ruta a través del Área de la Gran Bahía de China: oportunidades para América Latina

Daniel Morales Ruvalcaba 
Dossier América Latina: la iniciativa china de la Franja y la Ruta de los habitantes de Guangdong, influido por su alejamiento de las capitales del norte los hace estar orgullosos del nivel de autarquía que poseyeron desde tiempos antiguos" (Di Masi, 2006, p. 66). Efectivamente, la conjugación de elementos socioculturales y geográficos en la zona permitió que el dinamismo económico y comercial de Guangdong se incrementara con el paso del tiempo hasta consolidarse como el mayor puerto comercial de toda China durante la Dinastía Tang. Esto no sólo atrajo una importante cantidad de migrantes del interior de China (especialmente de la etnia Han), sino también comerciantes y empresarios de todo el mundo.

Es a lo largo del siglo XVI y gran parte del siglo XVII que Guangdong amplió y consolidó sus nexos con el mundo. Debido a su dinamismo económico, la ciudad de Guangzhou (capital de Guangdong) y sus alrededores se convirtieron en un punto neurálgico para el comercio entre la población china y los mercantes europeos quienes, después de cruzar el océano Índico y navegar por el estrecho de Malaca, llegaban a las costas del mar de China Meridional. Fue tal la importancia de esta zona que varias potencias europeas, ansiosas por tener un mejor acceso al mercado chino, buscaron la creación de asentamientos coloniales en territorios aledaños a Guangzhou: Portugal lo hizo con Macao en 1557, Gran Bretaña exigió los territorios de Hong Kong después de la Primera Guerra del Opio (1839-1842) y Francia comenzó a arrendar Zhanjiang a partir de 1898. Por todo ello, el académico William Boltz nombró a Guangzhou, metafóricamente, como la Sevilla de China (Boltz, 1967).

Durante la época de la Revolución Comunista, las políticas de autosuficiencia económica restaron importancia a la vinculación de Guangdong con el mundo. No obstante, con las reformas implementadas por Deng Xiaoping hacia finales de los setenta del siglo XX, esta provincia "se convirtió en el terreno experimental de China [...] cuando su gobierno central decidió reformar el sistema económico y abrir sus puertas a los inversores extranjeros" (Li y Yang, 2003). A lo largo de estas décadas, Guangdong transitó de ser una economía esencialmente agrícola a una industrializada (1979-1984), a dotarse luego de una infraestructura amplia y eficiente (1984-1992), para avanzar después hacia la alta industrialización económica y el desarrollo del sector terciario (1992-2003). Desde la primera década del siglo XXI, los gobiernos nacional y provincial han realizado grandes esfuerzos para impulsar actividades intensivas en conocimiento a través de la creación de "parques de alta tecnología, zonas de desarrollo industrial y ciudades universitarias en las principales ciudades y zonas de desarrollo designadas para actividades basadas en el conocimiento, formando nuevas concentraciones de los sectores emergentes" (Cheng, Boerboom, Geertman y Hooimeijer, 2013, p. 264).

Gracias a ello, Guangdong no sólo posee hoy una economía diversa y dinámica que resulta un modelo para el resto de China (Maruya, 1991), sino que además representa la provincia de mayor tamaño demográfico y económico, pues, según las cifras de 2016, su producto interno bruto alcanzó los RMB¥7,95 billones, lo cual representa el $11 \%$ del PIB de China (PwC, 2017, p. 5), mien- 
tras que su población superaba ya los 110 millones de habitantes. Por tal motivo, si se pudiera hacer una comparación entre las provincias de China y los estados de Estados Unidos, se podría decir que Guangdong sería la California de China. Además, de la misma forma en que el Área de la Bahía de San Francisco es el principal núcleo productivo y de desarrollo para California, es posible señalar que el Área de la Gran Bahía Guangdong-Hong Kong-Macao (粤港澳大湾区) concentra el mayor potencial de Guangdong.

El Área de la Gran Bahía, zona que comprende una superficie territorial de 56.000 kilómetros cuadrados y cuenta con una población de casi 70 millones de personas, reúne más del $80 \%$ de la manufactura industrial de Guangdong y recibe un promedio anual de 400 millones de turistas (domésticos e internacionales).

Para comprender mejor su composición, es necesario explicar que el AGB está integrada por 11 ciudades (ver Mapa 1): nueve ciudades se encuentran en China continental y dos corresponden a las Regiones Administrativas Especiales (RAE). Según su importancia económica, dichas ciudades son catalogadas de la siguiente forma:

- Ciudades nivel 1: estas son Guangzhou, Shenzhen y Hong Kong (RAE). Todas ellas registraron en 2018 un producto interno bruto superior a RMB¥2,2 billones (PwC, 2019, p. 4), equivalente a más de US\$300.000 millones.

- Ciudades nivel 2: estas son Foshan y Dongguan, las cuales en 2018 reportaron un producto interno bruto de entre RMB¥993.600 millones y 827.900

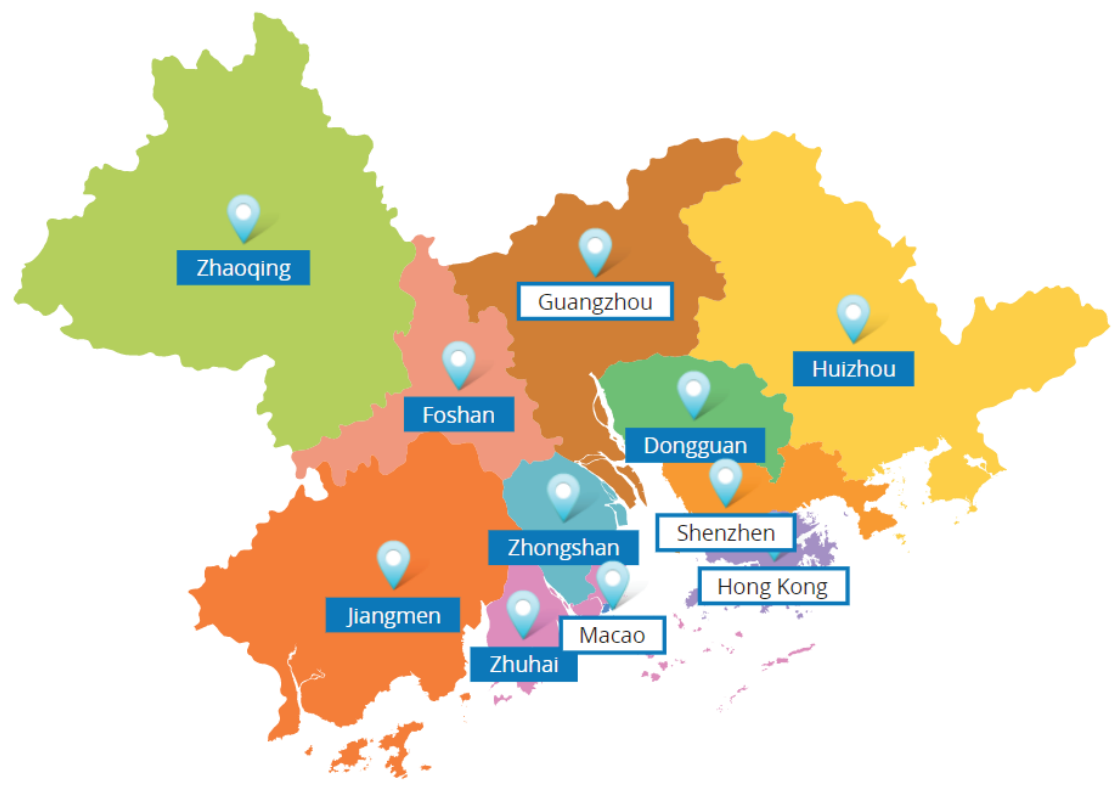

Fuente: Constitutional and Mainland Affairs Bureau, 2018.
Construcción de la Franja y la Ruta a través del Área de la Gran Bahía de China: oportunidades para América Latina

Daniel Morales Ruvalcaba

Mapa 1.

Ciudades del Área de la Gran Bahía Guangdong-Hong Kong-Macao 
Dossier América Latina: la iniciativa china de la Franja y la Ruta millones (PwC, 2019, p. 4), es decir, entre US\$150.000 millones y 120.000 millones, respectivamente.

- Ciudades nivel 3: a partir de su tamaño económico, el orden es Huizhou, Zhongshan, Macao (RAE), Jiangmen, Zhuhai y Zhaoqing. El PIB de todas ellas se coloca en un rango de entre RMB 410.300 millones (Huizhou) y RMB¥220.200 millones (Zhaoqing) (PwC, 2019, p. 4), esto es un rango equivalente a US\$60.000 millones y US\$30.000 millones, aproximadamente.

\section{Evolución y situación actual del Área de la Gran Bahía}

La idea del AGB tiene sus orígenes en el ámbito académico, específicamente en el seno de la Hong Kong University of Science and Technology (HKUST). Teniendo como inspiración la Bahía de San Francisco, fue en la segunda mitad de la década de los noventa cuando el profesor Chia-Wei Woo-entonces presidente de la HKUST- propuso la construcción de la Hong Kong Bay Area (HKBA). Lo relevante de la idea del profesor Woo es que su concepto no refería a una entidad política delimitada como Hong Kong, sino que proyectaba un área que, de ser impulsada de manera amplia, sería "un lugar que destaca por su belleza escénica y por un entorno idílico que, sin embargo, sustenta el crecimiento de un centro nacional e internacional de cultura, educación, finanzas, ciencia, alta tecnología, telecomunicaciones, transporte y turismo, así como otros servicios avanzados e industrias manufactureras" (Woo, 1998, p. 2). Gracias a su activismo desde el ámbito universitario, el profesor Woo ha sido considerado como el pionero y uno de los principales líderes de la conformación del Área de la Gran Bahía Guangdong-Hong Kong-Macao.

Ahora bien, como se puede apreciar en el Mapa 2, el proyecto de Woo situaba como puntos estratégicos las ciudades de Shenzhen, Nansha y Zhuhai, para conformar con ello un triángulo equilátero en torno al delta del río Perla, que funcionaría como una extensión natural y complementaria a Hong Kong. En ese sentido, cabe anotar que esta propuesta resultaba: 1) abarcadora, pues podía extenderse geográficamente en distintas direcciones; 2) multidimensional, pues no se limitaba a lo económico, sino que buscaba impulsar todos los ámbitos de la actividad humana; y 3) basada en el conocimiento, pues posicionaba a la universidad como la base para desarrollo económico, comercial y tecnológico de esta área.

La idea de Chia-Wei Woo tuvo gran impacto entre las autoridades y empresarios de China continental, especialmente de Guangdong. Cuando se propuso el concepto del HKBA en la década de los noventa, las economías de Guangzhou y Shenzhen -las dos ciudades más importantes de la Provincia de Guangdong- apenas comenzaban a despuntar. No obstante, frente a estas, el tamaño de Hong Kong era abrumador: la economía hongkonesa representaba, a mediados de la década de los noventa, alrededor de $1 / 4$ del producto 


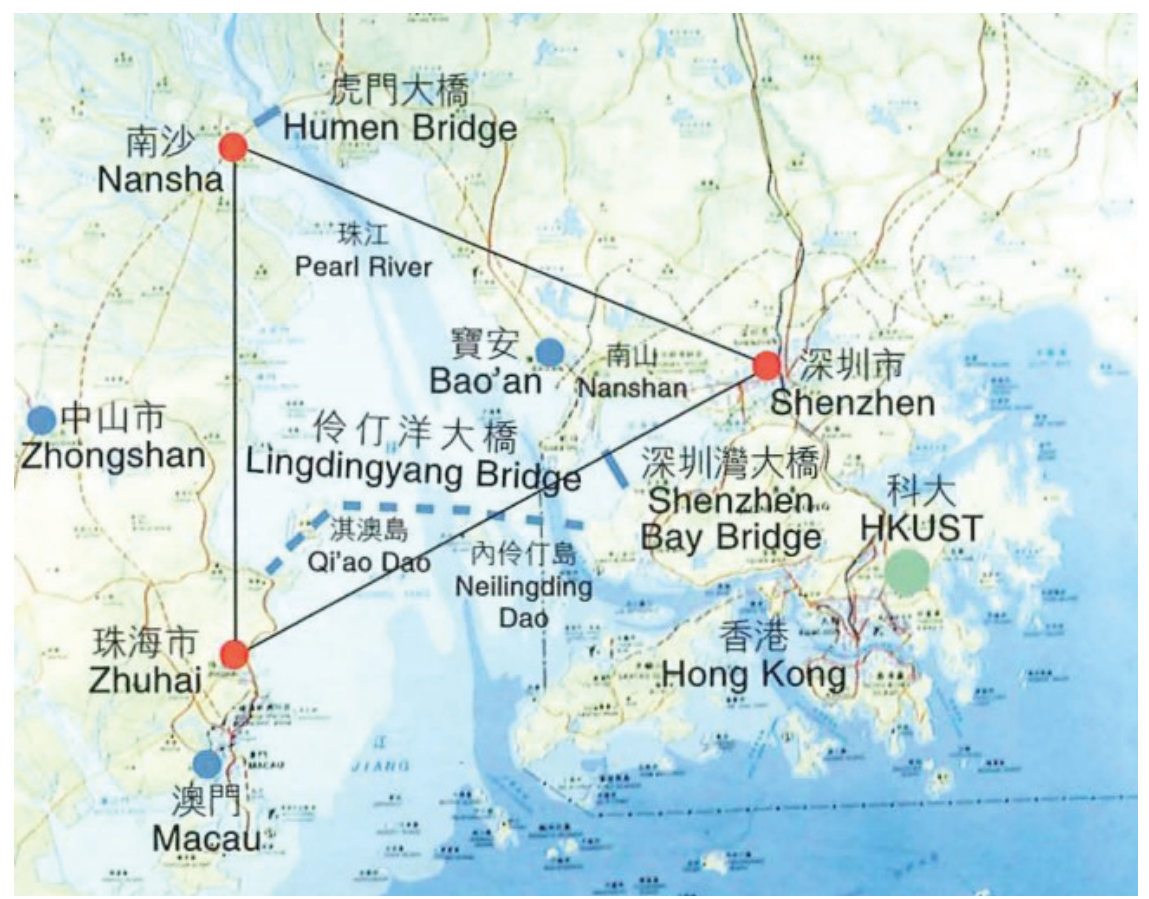

Fuente: Woo, 1998, p. 2.

interno bruto de toda China. Por ello, el gobierno cantonés consideró de gran importancia avanzar hacia la integración física y productiva de las ciudades chinas del delta del río Perla con Hong Kong, aunque "no habían podido tomar muchas iniciativas por sí mismos desde 1997 porque podría verse como una intervención en los asuntos internos de Hong Kong" (Yang, 2005, p. 2153).

A pesar de las complejidades jurídicas y administrativas, las autoridades chinas lograron, durante las últimas décadas del siglo XX y la primera del siglo XXI, que Hong Kong transfiriera una gran cantidad de fondos e industrias tradicionales a las ciudades vecinas del delta del río Perla. De acuerdo con Sxunasxi Valencia y Raquel León, "las estadísticas provinciales de Guangdong indican que más del $70 \%$ de todos los acuerdos de IED firmados entre 1979 y el 2006 [fueron] con compañías de la región administrativa especial de Hong Kong. Aproximadamente dos tercios de todas las importaciones de Hong Kong desde China Continental [provinieron] de la provincia de Guangdong" (Valencia y León, 2019, p. 99).

En un esfuerzo por facilitar la comunicación y la cooperación entre las partes, fue creada en 1998 la Hong Kong-Guangdong Cooperation Joint Conference, una importante plataforma política de la cual resultó la firma del Closer Economic Partnership Arrangement (CEPA) entre Hong Kong y el gobierno central de la República Popular de China el 29 de junio de 2003.
Construcción de la Franja y la Ruta a través del Área de la Gran Bahía de China: oportunidades para América Latina

Daniel Morales Ruvalcaba

Mapa 2.

Área de la Bahía de Hong Kong 
Dossier América Latina: la iniciativa china de la Franja y la Ruta
Si bien es cierto que Hong Kong contribuyó significativamente al desarrollo inicial del AGB, a partir de 2003 -luego de la firma del CEPA- fue percibido un cambio en la forma de integrarse entre las partes, pues este acuerdo no sólo otorgaría acceso preferencial a los productos hongkoneses, sino que también brindaría facilidades a las empresas cantonesas para invertir en Hong Kong. Estos acontecimientos motivaron a las autoridades de Guangdong a retomar y dar un decidido impulso a la conformación de un plan para las localidades del río Perla: el nombre designado para este proyecto fue el de Pan-Pearl River Delta (Pan-PRD). Así, mientras que el Pan-PRD Forum tomó su propio curso hacia la conformación de una región subnacional (Yeung, 2005; Yeh y Xu, 2008; 2011a; Yeung, Jianfa y Shen, 2008; Chen, Suo y Ma, 2015), el AGB quedó limitada a un área más reducida, con dinámicas y características propias.

Un paso decisivo para la conformación del AGB fue dado en diciembre de 2008 -en el marco del $30^{\circ}$ aniversario de la reforma y la apertura de China- con la publicación de The Outline of the Plan for the Reform and Development of the Pearl River Delta (2008-2020) (The National Development and Reform Commission, 2008) un documento que no sólo estableció y unificó los objetivos de desarrollo para el delta del río Perla, sino que enmarcó este proyecto local en ciertas estrategias geoeconómicas más amplias y de más largo plazo, como son el Pan-PRD Forum a nivel subnacional y el mecanismo ASEAN+China a nivel internacional.

Es posible señalar que el Outline vino a mejorar los vínculos económicos entre las ciudades del área. No obstante, en términos de la coordinación política, lo que prevaleció hasta estos momentos fue una mayor colaboración entre Guangdong y Hong Kong que entre Guangdong y Macao, exhibiendo con ello el déficit de una coordinación conjunta. Por ello, en febrero de 2009 fue creado el Liaison and Coordinating Meeting of Hong Kong, Guangdong and Macao, para discutir la implementación del Outline y para generar mayores sinergias de trabajo entre todas las partes. Si bien este mecanismo se consideró transitorio, pues debería llevar a la instalación de una instancia formal para la gobernanza y coordinación política de la zona, contribuyó a alcanzar "una serie de consensos sobre temas de infraestructura, [así como] acuerdos de transporte para mejorar la conectividad, turismo y protección ambiental" (Chan, 2011, p. 138).

Poco después, con el objetivo de formular recomendaciones para la implementación de estrategias de alto nivel entre las ciudades del delta del río Perla, fue publicado el Planning Study on the Coordinated Development of the Greater Pearl River Delta Township. Este documento marcó un hito en la configuración institucional del AGB, pues fue el primer estudio elaborado de manera conjunta por las autoridades de Guangdong, Hong Kong y Macao. Además, representó un adelanto teórico en la materia al concebir esta zona como una ciudad-región: "La exitosa cooperación entre Guangdong, Hong Kong y Macao en los últimos 30 años ha creado una ciudad-región que es una de las más singulares del mundo. Al coordinar el desarrollo, la región ha 
tratado y continuará lidiando con numerosos asuntos complicados" (Guangdong Province - Hong Kong SAR - Macao SAR, 2009, p. 3). De esta manera, el Planning Study estableció una importante pauta para la construcción de un área metropolitana de clase mundial que cubriría once ciudades instaladas en el delta del río Perla.

Es posible señalar que, hacia finales de la primera década del siglo XXI, el proyecto del AGB se encontraba en marcha, pero funcionando a nivel provincial y con un incipiente involucramiento de las autoridades nacionales.

\section{El AGB, una zona estratégica en la apertura de China y en la iniciativa de la Franja y la Ruta}

Un cambio significativo de conducir la gobernanza y coordinación de la zona correspondiente al delta del río Perla ocurrió cuando este proyecto fue incorporado en los planes quinquenales de desarrollo económico y social de la República Popular de China. Aún sin el nombre oficial de Área de la Gran Bahía de Guangdong-Hong Kong-Macao, esta zona comenzó a ser considerada como fundamental para la construcción de "una patria común para la nación china" por el estrecho vínculo entre China continental y sus RAE.

Si bien el $11^{\circ}$ Plan Quinquenal de China (2006-2010) fue descrito como uno "revolucionario, un punto de inflexión y de importancia decisiva" (Fan, 2006, p. 708), este no hizo mención alguna al delta del río Perla. Fue hasta el $12^{\circ}$ Plan Quinquenal (2011-2015) -el cual tuvo como principales objetivos reestructurar la economía china y mejorar los estándares de vida de su pueblo- que fue explícito el interés por profundizar los acuerdos marcos de cooperación de Guangdong con Hong Kong y Macao, como parte de la estrategia para la promoción del desarrollo regional. En este sentido, se propuso "fortalecer la planificación y coordinación, [y] mejorar el sistema de transporte entre el delta del río Perla y Hong Kong / Macao" (Central Committee of the Communist Party of China, 2011, p. XIV, c. 57).

Esta mención del delta del río Perla en los planes quinquenales no sólo fue retomada años después, sino que tuvo mayor impulso y relevancia. Así en el $13^{\circ}$ Plan Quinquenal (2016-2020), ya no sólo se subrayó el importante papel desempeñado por Hong Kong y Macao en la cooperación regional del delta del río Perla, sino que además se escribió: "Apoyaremos los esfuerzos conjuntos de Guangdong, Hong Kong y Macao para construir un área habitable de calidad en la región del Gran Delta del río Perla y acelerar el desarrollo de Qianhai, Nansha y Hengqin como plataformas de cooperación entre Guangdong, Hong Kong y Macao" (Central Committee of the Communist Party of China, 2016, p. XII, c. 54).

Con este ánimo, al año siguiente de la publicación del $13^{\circ}$ Plan Quinquenal, hubo dos acontecimiento decisivos para la conformación del AGB: el 5 de marzo, el primer ministro Li anunció que "se publicará y ejecutará el pro-
Construcción de la Franja y la Ruta a través del Área de la Gran Bahía de China: oportunidades para América Latina

Daniel Morales Ruvalcaba 
Dossier América Latina: la iniciativa china de la Franja y la Ruta grama de planificación del desarrollo de la gran bahía de Guangdong-Hong Kong-Macao, y se promoverá en todos los aspectos la cooperación mutuamente beneficiosa entre el interior y Hong Kong y Macao" (Keqiang, 2018); y, el 1 de julio, el presidente Xi junto con los jefes ejecutivos de Hong Kong y Macao, el gobernador de la Provincia de Cantón y el director de la Comisión Nacional de Desarrollo y Reformas de China firmaron el Framework Agreement on Deepening Guangdong-Hong Kong-Macao Cooperation in the Development of the Greater Bay Area (NDRC - Guangdong Province - Hong Kong SAR - Macao SAR, 2017). Con todo esto, el AGB se consolidaría como un espacio que, utilizando las ventanas propias de esas tres regiones, impulsaría el desarrollo y la apertura de la economía china en su conjunto.

La culminación del AGB ocurrió finalmente el 18 de febrero de 2019 cuando fue publicado el Outline Development Plan for the Guangdong-Hong KongMacao Greater Bay Area. Con este último documento, las autoridades chinas se propusieron construir no sólo una de las ciudades/región más productivas del mundo, sino también crear una zona que, para 2035, “debería convertirse en un sistema económico y un modo de desarrollo apoyado principalmente por la innovación, con sus fortalezas económicas y tecnológicas incrementadas enormemente y su competitividad e influencia internacional fortalecidas aún más" (NDRC - Guangdong Province - Hong Kong SAR - Macao SAR, 2019 , p. 10). Con todo ello, el AGB quedaría además vinculada a la iniciativa de la Franja y la Ruta a través del desarrollo de infraestructura, pues en la zona fueron creados tres megaproyectos:

1. El Enlace Ferroviario Express de Guangzhou - Shenzhen - Hong Kong, también conocido como "GuangShenGang XRL", que fue completado y puesto plenamente en funcionamiento en septiembre de 2018 para conectar a Hong Kong con la línea de ferrocarriles de alta velocidad de toda China (vía Shenzhen).

2. El Puente Hong Kong-Zhuhai-Macao (Puente HKZM), el cual entró en funcionamiento en octubre de 2018 y no sólo conecta a Zhuhai con las dos regiones administrativas especiales, sino que además reduce significativamente el tiempo de traslado entre las tres ciudades, generando además beneficios de desempeño logístico para el AGB (Vaz Pimentel, 2012, pp. 133-137; Valencia y León, 2019).

3. El Corredor Shenzhen-Zhongshan, que se espera sea concluido en 2024 y que reducirá el tiempo de traslado entre las dos orillas del río Perla a tan sólo 30 minutos.

Sin duda, el pleno funcionamiento de estos tres megaproyectos de infraestructura marcará una nueva era para el AGB, pues facilitará la internacionalización de empresas y capitales e incrementará aún más la competitividad de esta ciudad/región al incrementar su participación en las cadenas globales de valor. Lo más relevante es que el AGB quedará articulada a la red de proyectos de infraestructura que se impulsan desde la iniciativa de la Franja y la Ruta ya que, debido a su posicionamiento geoestratégico, el AGB está llamada 
a convertirse en el centro natural para la sección que vincula marítimamente a China con la cuenca del Pacífico y, desde ahí, América Latina.

\section{Oportunidades para el desarrollo de los intercambios sino-latinoamericanos desde el AGB}

Desde el AGB se han tejido importantes lazos entre China y América Latina a lo largo de la historia. Como un caso importante, se puede mencionar aquí a Macao. Cuando Macao alcanzó sus años de esplendor entre 1560 y 1640, fungió como un importante nodo en el comercio intra-asiático a través de la ruta Guangdong-Macao-Nagazaki, en el comercio euroasiático con la ruta Lisboa-Malaca-Goa-Macao y en el comercio asiático-americano en la ruta México-Manila-Macao-Guangdong. Una muestra de los intensos intercambios que ocurrieron desde Macao y Guangdong con América Latina se encuentra en las monedas de aquella época, pues "durante el siglo XVI se generalizó el uso de los pesos mexicanos en los puertos de la costa meridional de China, donde se empleaban para el tráfico del té y la seda a través de Manila” (McMaster, 1959, p. 376).

Actualmente, el AGB desempeña un papel relevante en las relaciones internacionales de China con América Latina.

Desde el punto comercial, el volumen de exportaciones de Guangdong hacia los países latinoamericanos alcanzó los US\$1.336 millones en los cuatro primeros meses de 2019, siendo los principales socios comerciales México, Brasil y Chile (Xinhua, 2019).

Desde el punto de vista social, es notable el impulso que se ha venido dando en el AGB al estudio y difusión de América Latina. De acuerdo con el informe de Myers, Barrios y Guo (2018) cuatro centros de investigación especializados en la región se encuentran operando en la bahía: 1) Latin America Research Center (Guangdong University of Foreign Studies), 2) Center for Latin American Studies (Jinan University), 3) Institute for Research on Portuguese-Speaking Countries (City University of Macau), 4) Center for Latin American Studies (Sun Yat-sen University). Se espera que con la extensión de la iniciativa de la Franja y la Ruta hacia América Latina se promueva aún más el desarrollo de los estudios latinoamericanos en China.

Desde el punto de vista geográfico, el AGB juega un rol destacado y, dentro de dicha área, la ciudad de Zhuhai se presenta como una oportunidad valiosa. Con un área de $1.736 .5 \mathrm{~km}^{2}$ y una población de 1.891 .000 en 2018 (pero que se proyecta en 5 millones para 2030), Zhuhai se encuentra localizada al sur de la costa oeste de la delta del río Perla (ver Mapa 1 y Mapa 2). Aquí ocupa un lugar muy estratégico: por un lado, Zhuhai cuenta con 7 puertos, 2 autopistas de la red nacional, 2 líneas de transporte férreo y 5 aeropuertos en un radio de $150 \mathrm{~km}$, todo lo cual vincula a esta ciudad con el resto del país. Además, en octubre de 2018 Zhuhai pasó a convertirse en la única ciudad conectada
Construcción de la Franja y la Ruta a través del Área de la Gran Bahía de China: oportunidades para América Latina

Daniel Morales Ruvalcaba 
Dossier América Latina: la iniciativa china de la Franja y la Ruta directamente con las dos regiones administrativas especiales de China gracias a la puesta en funcionamiento del Puente Hong Kong-Zhuhai-Macao.

Todo ello se encuentra potenciado, por un lado, gracias al estatus de Zona Económica Especial que Zhuhai goza desde 1979 y, por otro, a la participación de esta ciudad en el GuangDong Free Trade Zones (GDFTZ), un triángulo ${ }^{1}$ de zonas económicas especiales pensadas como un programa piloto para la apertura de China.

Desde el punto de vista político, es posible señalar que, en la segunda década del siglo XXI, Zhuhai asumió un claro liderazgo para el establecimiento de lazos con América Latina, realizando eventos para la promoción de negocios, inversiones, cultura, deportes y turismo, entre los cuales hay que destacar: la Exposición Internacional China-América Latina y el Caribe, realizada del 9 al 11 de noviembre de 2017 y patrocinada por la Cámara de Comercio Internacional de China; y la celebración de la XII Cumbre Empresarial China-América Latina (CECAL), del 1 al 3 de noviembre de 2018 en Zhuhai. No es casualidad que Zhuhai fuera seleccionada para albergar ambos eventos, pues, desde finales de 2017, se ha construido en dicha ciudad el "Parque de Cooperación Económica y Comercial entre China y América Latina y el Caribe” en el Distrito de Hengqin, que cuenta con 244.000 metros cuadrados y supera un costo de RMB¥2.500 millones (Xinhua Español, 2017), que representa más de US\$350 millones.

El Parque de Cooperación de Hengqin no sólo estará exento de impuestos para la entrada de bienes latinoamericanos, sino que también ofrecerá políticas preferenciales, telecomunicaciones de alta velocidad, incentivos fiscales y financieros para facilitar la estancia de compañías de América Latina en China e impulsar con ello las relaciones económicas entre las partes. Al respecto Mariano Mosquera ha encontrado que "en Hengqin la dialéctica es informal -cultural- y es la coordinación corporativa a posteriori la que transforma el fenómeno creativo en un proceso de política pública [donde] la 'combinación creativa' (R.L.S.) funciona como una narrativa que relaciona el momento dialéctico con la innovación, mientras que la coordinación -y sus condiciones de credibilidad- ejerce de mecanismo causal para relacionar el corporativismo con las políticas públicas" (Mosquera, 2020, p. 344). Todo ello indica que en Hengqin se están desarrollando condiciones extraordinarias para la innovación en políticas públicas mismas que tienen, como uno de sus principales objetivos, detonar las relaciones económicas entre China y América Latina.

En síntesis, es posible afirmar que el AGB ha asumido el liderazgo en el establecimiento de lazos con América Latina, realizando eventos y construyendo infraestructura para la promoción de negocios, inversiones, cultura,

1 Las GDFTZ fueron aprobadas y establecidas por el Consejo de Estado el 31 de diciembre de 2014 (Guangdong Foreign Economic Relations \& Trade, 2015). Estas cubren un total de 116,2 km2 que se encuentran distribuidas en 3 polos: el área de Nanzha en Guangzhou (con $60 \mathrm{~km} 2$ ), el área de Qianhai y Shekou en Shenzhen (con 28,2 km2) y el área de Hengqin en Zhuhai (con $28,2 \mathrm{~km} 2$ ). 
deportes y turismo. Resta ahora observar de qué manera América Latina gestiona su entrada a China, en la misma que ahora se abre una puerta desde el Área de la Gran Bahía de Guangdong-Hong Kong-Macao.

\section{Referencias}

Boltz, W. (1967). Canton: the Seville of China. Romance Philology, 21(2), pp. 171-174. Recuperado el noviembre de 2019, de https://www.jstor.org/stable/44940338

Bui, T., Jones, W., Li, J. y Yang, D. (2003). Preface. En T. Bui, D. Yang, W. Jones y J. Li, China's economic powerhouse: reform in Guangdong Province (pp. ix-xiii). New York: Palgrave Macmillan. doi:10.1057/9780230508668

Central Committee of the Communist Party of China. (2011). Outline of the 12th FiveYear Plan for National Economic and Social Development of the People's Republic of China. Recuperado el noviembre de 2019, de European Commission: https://ec.europa.eu/ regional_policy/sources/international/pdf/china_draft_12th_5yearplan17032011.pdf

Central Committee of the Communist Party of China. (2016). The 13th Five-Year Plan for Economic and Social Development of the People's Republic of China, 2016-2020. Beijing: Central Compilation \& Translation Press.

Chan, R. (2011). Hong Kong's Position in Guangdong under "One Country, Two Systems". Seoul Journal of Economics, 24(2), pp. 125-150.

Chen, B., Suo, L. y Ma, J. (2015). A Network Approach to Interprovincial Agreements: A Study of Pan Pearl River Delta in China. State \& Local Government Review, 47(3), pp. 181-191. Recuperado el noviembre 2019, de https://www.jstor.org/stable/24639234

Cheng, F., Boerboom, L., Geertman, S. y Hooimeijer, P. (2013). Changing spatial concentration of sectoral employment in China's Pearl River Delta 1990-2005. Tijdschrift voor Economische en Sociale Geografie, 104(3), pp. 261-277. doi:10.1111/j.1467-9663.2012.00741.x

Constitutional and Mainland Affairs Bureau. (2018). Greater Bay Area. Recuperado el noviembre de 2019, de https://www.bayarea.gov.hk/en/home/index.html

Di Masi, J. (2006). El delta del Río Perlas, la integración regional y el desarrollo económico chino. Estudios Internacionales, 38(152), pp. 63-73. doi:10.5354/0719-3769.2011.14366

Fan, C. (2006). China's Eleventh Five-Year Plan (2006-2010): From "Getting Rich First" to "Common Prosperity". Eurasian Geography and Economics, 47(6), pp. 708-723. doi:10.2747/1538-7216.47.6.708

Guangdong Foreign Economic Relations \& Trade. (2015). 中国 (广东) 自由贸易试验区. Recuperado el noviembre de 2019, de China (Guangdong) Pilot Free Trade Zone: https:// bit.ly/2OKolKP

Guangdong Province - Hong Kong SAR - Macao SAR. (2009). Building Coordinated and Sustainable World-class City-region. Planning Study on the Coordinated Development of the Greater Pear River Delta Townships. Recuperado el noviembre de 2019, de Direcção dos Serviços de Solos, Obras Públicas e Transportes da RAEM: https://urbanplanning.dssopt. gov.mo/pt/download/zhu02.pdf

Keqiang, L. (2018). Informe sobre la Labor del Gobierno. Recuperado el noviembre de 2019, de Observatorio de la Política China: https://politica-china.org/secciones/texto-integroinforme-sobre-la-labor-del-gobierno
Construcción de la Franja y la Ruta a través del Área de la Gran Bahía de China: oportunidades para América Latina

Daniel Morales Ruvalcaba 
Dossier América Latina: la iniciativa china de la Franja y la Ruta
Li, J. y Yang, D. (2003). Guangdong: China's Economic Powerhouse - The Past, the Present and the Future.En T.Bui, D. Yang, W. JonesyJ.Li, China'seconomicpowerhouse: reform in Guangdong Province (pp. 208-231). New York: Palgrave Macmillan. doi:10.1057/9780230508668

Maruya, T. (1991). Guangdong as a regional model of economic development in China. En E. Chen y T. Maruya, A Decade of "Open-Door" Economic Development in China, 19791989 (pp. 153-183). Tokyo: Institute of Developing Economies.

McMaster, J. (1959). Aventuras asiáticas del peso mexicano. Historia Mexicana, 8(3), pp. 372-399.

Mosquera, M. (2020). Incrementalismo dialéctico. Un caso de innovación en Hengqin (China). Gestión y Política Pública, XXIX(2), pp. 321-353. doi:10.29265/gypp.v29i2.776

Myers, M., Barrios, R. y Guo, C. (junio de 2018). Learning Latin America: China's Strategy for Area Studies Development. Recuperado el 2018, de The Dialogue: https://bit.ly/2Ijsm42

NDRC - Guangdong Province - Hong Kong SAR - Macao SAR. (2017). Framework Agreement on Deepening Guangdong-Hong Kong-Macao Cooperation in the Development of the Bay Area. Recuperado el noviembre de 2019, de Greater Bay Area: https://www.bayarea. gov.hk/filemanager/en/share/pdf/Framework_Agreement.pdf

NDRC - Guangdong Province - Hong Kong SAR - Macao SAR. (2019). Outline Development Plan for the Guangdong-Hong Kong-Macao Greater Bay Area. Recuperado el noviembre de 2019, de Greater Bay Area: https://www.bayarea.gov.hk/filemanager/en/ share/pdf/Outline_Development_Plan.pdf

PwC. (2017). New Opportunities for Guangdong-Hong Kong-Macau Greater Bay Area. Recuperado el octubre de 2019, de PwC China: https://pwc.to/2nsYlZK

PwC. (2019). PwC's Report on cities of the Guangdong-Hong Kong-Macao Greater Bay Area: To develop new growth potential in the Guangdong-Hong Kong-Macao GBA: Dongguan. Recuperado el noviembre de 2019, de PwC China: https://www.pwccn.com/en/researchand-insights/greater-bay-area-economic-growth-dongguan.pdf

The National Development and Reform Commission. (2008). The Outline of the Plan for the Reform and Development of the Pearl River Delta (2008-2020). Recuperado el noviembre de 2019, de Office of the Provost: https://bit.ly/2DHOAvg

Valencia, S. y León, R. (2019). Infraestructura y competitividad en la Iniciativa de la Franja y la Ruta: implicaciones geoeconómicas en el caso del puente HZM. Razón Crítica(7), pp. 81-110. doi:10.21789/25007807.1499

Vaz Pimentel, M. (2012). How to sell Macau. Learning with the territory's staged urban reinvention. Coimbra: University of Coimbra.

Woo, C.-W. (1998). The Hong Kong Bay Area-A Metropolis for the 21st Century. Recuperado de The Hong Kong University of Science and Technology. Recuperado el noviembre de 2020, de https://archives.ust.hk/dspace/bitstream/9999/16933/1/pao-01b-a035.pdf

Xinhua. (2019). Guangdong sees import and export with Latin American countries up 10 pct in Jan.-April. Recuperado el noviembre de 2019, de Xinhuanet: http://www.xinhuanet. com/english/2019-05/31/c_138105312.htm

Xinhua Español. (2017). Inauguran Parque de Cooperación Económica y Comercial ChinaLAC en Zhuhai. Recuperado el noviembre de 2020, de Xinhuanet: http://spanish.xinhuanet. com/2017-11/10/c_136743218.htm

Yang, C. (2005). Multilevel governance in the cross-boundary region of Hong Kong - Pearl River Delta, China. Environment and Planning, 37(12), pp. 2147-2168. doi:10.1068/a37230 
Yeh, A. y Xu, J. (2008). Regional Cooperation in the Pan-Pearl River Delta: A Formulaic Aspiration or A New Imagination? Built Environment, 34(4), pp. 408-426. Recuperado de https://www.jstor.org/stable/23289850

Yeh, A. y Xu, J. (2011a). China's Pan-Pearl River Delta: Regional Cooperation and Development. Hong Kong: Hong Kong University Press. Recuperado de https://www.jstor. org/stable/j.ctt1xwcwr

Yeung, Y.-m. (2005). Emergence of the Pan-Pearl River Delta. Geografiska Annaler, 87(1), pp. 75-79. Recuperado de https://www.jstor.org/stable/3554446

Yeung, Y.-m., Jianfa, S. y Shen, J. (2008). The Pan-Pearl River Delta. An Emerging Regional Economy in a Globalizing China. Hong Kong: The Chinese University Press.
Construcción de la Franja y la Ruta a través del Área de la Gran Bahía de China: oportunidades para América Latina

Daniel Morales Ruvalcaba 